\title{
Previous hospitalizations predict both hospital readmissions and mortality in patients with heart failure
}

We read the article entitled "Prior hospital admission predicts thirty-day hospital readmission for heart failure patients" by McLaren et al. [1] with great interest. In this well designed analysis, the authors reported that hospital readmission is associated with increased risk for 30-day hospital readmission in heart failure (HF) patients.

Reducing the hospital readmission rates is quite important in patients with $\mathrm{HF}$ in regard to high cost conditions as well as patients prognosis. Of course, it is obvious that adherence to $\mathrm{HF}$ guidelines has clinical benefit, however it des not always translate into consistent reductions in readmissions. Prediction of hospital readmissions and prevention in these conditions are possible. Efforts made to predict the highest risks for readmission in HF patients are necessary in this context. In the present study, the investigators showed that the rate of a 30-day readmission increased with the number of prior hospital admissions. Furthermore, they showed that prior hospitalization improved the accuracy of readmission prediction model [1]. Identifying readmission predictors such as co-morbidities, frailty, or other demographics would be beneficial for patient prognosis and action of spending healthcare funds. For this purpose, several risk models for prediction of readmission in $\mathrm{HF}$ patients were developed [2, 3]. However, mostly the risk models have limited usefulness in clinical practice. It is also necessary to keep in mind that readmissions are generally unrelated to $\mathrm{HF}$, but non-cardiovascular co-morbidities have larger effect size in HF patients. Also, unmeasured factors in studies related to healthcare system or patient characteristics such as socioeconomic, health status, adherence, and psychosocial factors may affect the readmissions in this group of patients. Prior hospitalizations are not only related to readmission rates but also an independent predictor of mortality in patients with HF. Prior analyses of our group in 630 patients with severe systolic dysfunction with mean ejection fraction of $25 \pm 10 \%$ showed that number of previous hospitalizations is an independent risk factor for mortality as well as older age, having orthopnea, lack of renin-angiotensin system inhibitor therapy at discharge, hyperuricemia $(>7 \mathrm{mg} / \mathrm{dL}$ ) and low hemoglobin level $(<10 \mathrm{~g} / \mathrm{dL})$ [4]. In our cohort, $50 \%$ of the patients have 2 or more prior hospitalizations. Therefore, prior hospital admissions are also a mortality predictor especially in HF patients with severe systolic dysfunctions.

\section{Conflict of interest: None declared}

\section{References}

1. McLaren DP, Jones R, Plotnik R et al. Prior hospital admission predicts thirty-day hospital readmission for heart failure patients. Cardiol J, 2016; 23: 155-162. doi: 10.5603/CJ.a2016.0005.

2. Allen LA, Smoyer Tomic KE, Smith DM et al. Rates and predictors of 30-day readmission among commercially insured and Medicaid-enrolled patients hospitalized with systolic heart failure. Circulation Heart Failure, 2012; 5: 672-679.

3. Eapen ZJ, Liang L, Fonarow GC et al. Validated, electronic health record deployable prediction models for assessing patient risk of 30-day rehospitalization and mortality in older heart failure patients. J Am Coll Cardiol Heart Failure, 2013; 1: 245-251.

4. Tokatli A, Karauzum K, Ural D et al. Discharge risk scoring method for predicting mortality in hospitalized chronic heart failure patients with severe systolic dysfunction. Acta Cardiol, 2015; 70: 442-449.

Alptug Tokatli ${ }^{1}$, Dilek Ural $^{2}$

${ }^{1}$ Department of Cardiology, Golcuk Military Hospital, Golcuk-41910, Kocaeli, Turkey, tel: +90262 42602 71, fax: +90 026241411 11, e-mail:alptugtokatli@gmail.com ${ }^{2}$ Department of Cardiology, Kocaeli University, Kocaeli, Turkey 